\title{
Implementación del ultrasonido pulmonar en la UCI durante la pandemia de COVID-19
}

Implementation of lung ultrasound in the ICU during the COVID-19 pandemic Implementação do ultrassom pulmonar na UTI durante a pandemia de COVID-19

Francisco Godínez García, ${ }^{\star}$ Edgar Bravo Santibáñez, Diana Vega Martínez, ${ }^{*}$ Pedro Luis González Carrillo, Omar Israel Hernández Mejía, ${ }^{*}$ Salvador Domínguez Estrada*

\section{RESUMEN}

El surgimiento del nuevo coronavirus SARS-CoV-2 está desafiando seriamente a la comunidad médica en prácticamente todo el mundo. La neumonía causada por COVID-19 tiene características peculiares y puede estudiarse mediante ultrasonografía pulmonar. La ecografía permite identificar el comportamiento de la infección y su progresión al lado de la cama del paciente. La técnica ha evolucionado considerablemente en los últimos años en lo que respecta a sus aspectos teóricos y operativos. En consecuencia, su aplicación clínica ha llegado a ser suficientemente conocida y generalizada en el contexto de paciente críticamente enfermo. Sugerimos la identificación de cuatro patrones en el ultrasonido pulmonar, los cuales permiten una mejor caracterización de la enfermedad. Debido a la sobredemanda de los servicios de salud en la actualidad, el conocimiento y aplicación de la ecografía pulmonar resulta de gran relevancia. Esta revisión incluye un enfoque práctico y proporciona un resumen de la evidencia para el uso e interpretación del ultrasonido pulmonar.

Palabras clave: COVID-19, ultrasonido pulmonar, unidad de terapia intensiva.

\section{ABSTRACT}

The emergence of the novel coronavirus (SARS-CoV-2) is seriously challenging the medical community virtually the entire world. Pneumonia in COVID-19 has peculiar characteristics and can be studied by lung ultrasound. The ultrasound allows identifying the behavior of the infection and its progression at the bedside of the patient. The technique has evolved considerably in recent years in terms of its theoretical and operational aspects. Consequently, its clinical application has become sufficiently known and generalized in the context of the critically ill patient. We suggest the identification of 4 patterns on lung ultrasound which allow a better characterization of the disease. Due to the current demand for health services, the knowledge and application of lung ultrasound is highly relevant. This review includes a pragmatic approach and provides a summary of the evidence for the use and interpretation of lung ultrasound.

Keywords: COVID-19, lung ultrasonography, critical care.

\section{RESUMO}

O surgimento do novo coronavírus SARS-CoV-2 está desafiando seriamente a comunidade médica em praticamente todo o mundo. A pneumonia no COVID-19 tem características peculiares e pode ser estudada por ultrassom pulmonar. A ultrassonografia permite identificar o comportamento da infecção e sua progressão na beira do leito do paciente. A técnica evoluiu consideravelmente nos últimos anos em relação aos seus aspectos teóricos e operacionais. Consequentemente, sua aplicação clínica tornou-se suficientemente conhecida e generalizada no contexto do paciente crítico. Sugerimos a identificação de quatro padrões na ultrassonografia pulmonar, o que permite uma melhor caracterização da doença. Devido à demanda atual por serviços de saúde, o conhecimento e a aplicação da ultrassonografia pulmonar são altamente relevantes. Esta revisão inclui uma abordagem prática e fornece um resumo das evidências para o uso e a interpretação do ultrassom pulmonar.

Palavras-chave: COVID-19, ultrassom pulmonar, Unidade de Terapia Intensiva.

\section{INTRODUCCIÓN}

Debido a su alta transmisibilidad, la enfermedad por coronavirus 2019 (COVID-2019) se ha extendido rápi-

\footnotetext{
* Especialista en Medicina del Enfermo en estado crítico. Unidad de Cuidados Críticos del Hospital General de León.

Recepción: 05/05/2020. Aceptación: 22/05/2020.

www.medigraphic.com/medicinacritica
}

damente por todo el mundo. ${ }^{1}$ La patogenia del SARSCoV-2 sigue sin ser del todo clara; sin embargo, se sabe que el virus utiliza los receptores de enzima convertidora de angiotensina 2 (ACE-2) para invadir las células diana. Dichos receptores se encuentran ampliamente expresados en el epitelio pulmonar, intestinal, renal, miocardio y en el sistema circulatorio. ${ }^{2}$ Los pacientes frecuentemente presentan síntomas asociados con neumonía viral, tos seca, fiebre, mialgias y fatiga; ${ }^{3}$ aunque los hallazgos radiológicos son muy variables, sin embargo, en más de $75 \%$ de los pacientes se identifica lesión bilateral. ${ }^{4}$

En la tomografía de tórax lo más frecuente es identificar las áreas de infiltrado, las cuales se caracterizan por opacidades en «vidrio deslustrado» y, según algunos reportes, hasta $29 \%$ cursa con zonas francas de consolidación. ${ }^{5}$ Se reporta que la práctica de la ultrasonografía enfocada es una modalidad de imagen clínica cada vez más utilizada para fines de diagnóstico y monitoreo, y es de vital importancia en una serie de afecciones comunes de la Unidad de Cuidados Intensivos (UCI), incluido el síndrome de dificultad respiratoria aguda. ${ }^{6}$

\section{VENTAJAS DEL ULTRASONIDO PULMONAR SOBRE LA RADIOGRAFÍA Y TOMOGRAFÍA DE TÓRAX}

El ultrasonido pulmonar (USP) es una técnica de imagen que se realiza al pie de la cama del paciente en la Unidad de Cuidados Intensivos. Tradicionalmente, el abordaje de las patologías pulmonares en la $\mathrm{UCl}$ se ha basado en la radiografía de tórax anteroposterior y la tomografía axial computarizada (TAC). Esta última se considera la mejor forma de valoración pulmonar en el paciente crítico. La introducción del USP ha revolucionado la evaluación y monitoreo de los pacientes en la UCI moderna. ${ }^{7-9}$

La radiografía es de uso rutinario, pero afortunadamente cada vez menor; en la $\mathrm{UCl}$ se le asignan múltiples aplicaciones como la evaluación de la adecuada colocación de dispositivos invasivos (acceso vascular y tubo endotraqueal), así como parte del abordaje diagnóstico en la falla respiratoria; sin embargo, en un estudio deficiente (diferencias interobservador, técnica, poca sensibilidad y especificidad en las diferentes 
patologías), el USP, sin duda, tomará su lugar como la técnica rutinaria en la $\mathrm{UCl} .^{10}$

En el 2004, Lichtenstein y colaboradores compararon diferentes métodos diagnósticos como son: la parte clínica (auscultación), radiografía de tórax, y ultrasonido pulmonar. La radiografía tuvo una precisión diagnóstica de baja a media (47\% para derrame pleural, $75 \%$ para consolidación y $72 \%$ para síndrome alveolo-intersticial), en cambio el USP presentó una precisión diagnóstica alta (93\% para derrame pleural, 97\% para consolidación y $95 \%$ para síndrome alveolointersticial) y tuvo un rango de error interobservador de 0.74 y 0.78 .11

En el 2011, Xirouchaki y su equipo compararon la precisión diagnóstica del USP en contra de la radiografía de tórax para cuatro patologías (consolidación, síndrome intersticial, neumotórax y derrame pleural). Ellos encontraron mejor poder diagnóstico del USP con una media de sensibilidad y especificidad alta (94 y 92\%) $\mathrm{y}$, en cambio, de la radiografía de tórax muy baja (55 y $62 \%) .{ }^{12}$

M. Winkler y su equipo realizaron un metaanálisis sobre la exactitud diagnóstica de la radiografía de tórax contra la ecografía pulmonar en pacientes críticos; ellos encontraron que la sensibilidad y especificidad de la radiografía de tórax es de 49 y $92 \%$, respectivamente; por otro lado, la ecografía de tórax presentó una sensibilidad de $95 \%$ y una especificidad de $94 \%$. $^{13}$

Se resume la sensibilidad y especificidad del USP para las cinco patologías pulmonares más comunes en la Unidad de Cuidados Intensivos en la Tabla 1.

La tomografía es el estándar de oro para la evaluación pulmonar por imagen en el paciente crítico. Tiene una muy alta sensibilidad y especificidad para la detección de diferentes patologías (por ejemplo, para derrame pleural tiene una sensibilidad de $100 \%$ y una especificidad de 99.7\%). Sin embargo, para el paciente crítico la tomografía tiene múltiples contras, por ejemplo: es un estudio estático (el ultrasonido es una evaluación dinámica), el traslado para la realización

Tabla 1: Sensibilidad y especificidad del ultrasonido pulmonar en diferentes patologías.

\begin{tabular}{lcc}
\hline & Sensibilidad (\%) & Especificidad (\%) \\
\hline $\begin{array}{l}\text { Derrame pleural (Intensive Care Med. } \\
\text { 25:955-958) }\end{array}$ & 94 & 97 \\
$\begin{array}{l}\text { Consolidación pulmonar (Intensive Care } \\
\text { Med. 30:276-281) }\end{array}$ & 90 & 98 \\
$\begin{array}{l}\text { Síndrome intersticial (Am J Respir Crit } \\
\text { Care Med. 156:1640-1646) }\end{array}$ & 93 & 93 \\
$\begin{array}{l}\text { Neumotórax completo (Intensive Care } \\
\text { Med. 25:383-388) }\end{array}$ & 100 & 96 \\
Neumotórax oculto (Crit Care Med. & 79 & 100 \\
33:1231-1238) & & \\
\hline
\end{tabular}

Modificada de: Lichtenstein DA. Lung ultrasound in the critically ill: the BLUE protocol. New York: Springer Science; 2016. de la TAC puede provocar daños y complicaciones al paciente (retiro accidental de acceso venoso central, tubo endotraqueal, sondas, hipoxemia, hipotensión) y se debe agregar la exposición a la radiación (7 mSv de radiación efectiva frente a $0.1 \mathrm{mSv}$ de la radiografía de tórax). ${ }^{14}$

Nazerian y colaboradores, en 2014, compararon el USP contra la TAC de tórax para evaluar la exactitud diagnóstica de las consolidaciones pulmonares; encontraron que, en comparación con la tomografía de tórax, el USP tiene una sensibilidad de 82 a $91 \%$ y una especificidad de 95 a $97 \%$ sin ser superior a la tomografía de tórax, pero con la ventaja de ser una herramienta confiable para realizar el diagnóstico de consolidaciones pulmonares al pie de la cama del paciente. ${ }^{15}$

Tierney y su equipo, en 2020 , en un estudio prospectivo en el que el objetivo fue evaluar el ultrasonido pulmonar de nueve puntos, sumado a una radiografía frente a TAC de tórax para localizar la patología pulmonar, no encontraron que el USP fuera superior a la TAC de tórax, pero sí muy similar, pues hubo una correlación positiva de 0.87 entre la TAC de tórax y el USG pulmonar. ${ }^{16}$ El protocolo LUCI-FLR ( $A$ Bedside Alternative to Irradiating Techniques, Radiographs and $C T)$ se creó como respuesta a la exposición repetitiva de radiación por estudios de imagen, tales como la radiografía de tórax y la tomografía. Al considerar que éstas iban en aumento en la Unidad de Cuidados Intensivos, el objetivo del protocolo fue y sigue siendo disminuir en un tercio, dentro de las tres próximas décadas, las radiografías a la cabecera del paciente y reducir las tomografías en dos tercios («L» de limitar), ${ }^{17}$ pues una de sus grandes aplicaciones es la detección de neumotórax (uso del USP como estándar de oro antes que la tomografía, y sólo realizarla en casos de neumotórax oculto).

Por lo anterior, destacamos la importancia en la implementación de la ultrasonografía pulmonar como elemento de diagnóstico y monitoreo indispensable para el manejo de la pandemia actual.

\section{APLICACIÓN DEL ULTRASONIDO PULMONAR EN EL CONTEXTO DE COVID-19}

Equipo. Se acepta que no es necesario un equipo de alta tecnología para la práctica de ultrasonografía pulmonar. ${ }^{18}$ En el contexto de la pandemia por COVID-19, los equipos más apropiados son aquéllos basados en sondas y transductores portátiles; estos dispositivos se pueden proteger fácilmente con cubiertas de plástico de un solo uso, lo que reduce el riesgo de contaminación y facilita los procedimientos de esterilización. Como esto no siempre es posible, se pueden emplear equipos de mayor tamaño, pero bajo la reserva de brindar un uso exclusivo. 


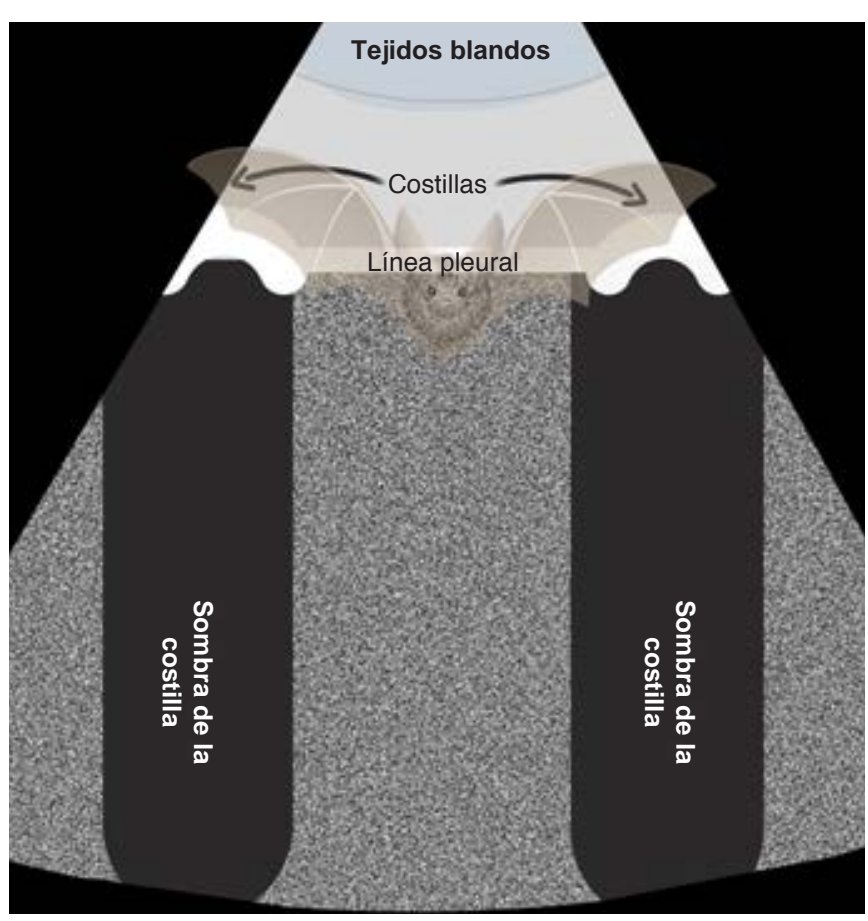

Figura 1: Signo del murciélago.

Podemos contemplar como requisitos mínimos los siguientes:

1. Equipo portátil, aparato de tamaño pequeño, calidad de imagen óptima, de rápido encendido, fácil transporte y costo accesible.

2. El tipo de transductor adecuado es aquél que trabaje en un rango de frecuencia entre $2-10 \mathrm{MHz}$ (convexo, microconvexo y/o lineal) y permita la visualización de imágenes en un rango de $1-12 \mathrm{~cm}$.

3. Tecnología de imagen que permita la visualización 2-D (bidimensional) y M-Mode.

Regiones anatómicas a explorar. Existen múltiples divisiones anatómicas para el abordaje del tórax, muchas de las cuales obedecen incluso a preferencias de los investigadores. Se han desarrollado múltiples técnicas de exploración, algunas aplicadas a contextos particulares, por ejemplo, la rápida identificación en emergencia respiratoria (protocolo BLUE), así como la visualización y búsqueda detallada de 28 espacios intercostales. Para fines prácticos, nosotros sugerimos una exploración breve con una técnica orientada a la visualización de 12 espacios, seis espacios por pulmón. La técnica sugerida en el consenso del 2012. ${ }^{19,20}$

Los principales síntomas relacionados con COVID-19 son: fiebre, tos y dificultad respiratoria, con un amplio espectro de severidad. ${ }^{2,3}$ Se establece que $80 \%$ de los pacientes tiene síntomas leves, pero aproximadamente $15 \%$ experimenta enfermedad moderada-severa y $5 \%$ desarrolla una enfermedad crítica. ${ }^{21}$ En un porcentaje de $20 \%$ de los pacientes que contraen COVID-19 y que requieren hospitalización, la evolución tomográfica y ultrasonográfica pulmonar es típicamente progresiva. En el transcurso de esta progresión, los cambios en el parénquima comienzan en las regiones distales de pulmón. Hay opacidades en «vidrio deslustrado» y predominio de consolidaciones en la región posterobasal. ${ }^{22}$

Aunque existen varios signos para la correcta identificación de los distintos síndromes ultrasonográficos pulmonares, nos centraremos en la evolución hasta el momento descrita del comportamiento en COVID-19. ${ }^{23}$ Antes de ello mencionaremos los signos básicos, los cuales integraremos posteriormente en síndromes.

Signo del murciélago. La localización de esta imagen es un paso fundamental en la valoración del pulmón. Al colocar el transductor en sentido vertical, se observa una imagen compuesta por las sombras acústicas de las costillas, los tejidos blandos en la porción superior, la línea pleural y los artefactos que emergen de ella (Figura 1).

Pleura o línea pleura: es la línea hiperecogénica localizada entre las sombras acústicas de las estructuras óseas y se encuentra por debajo de los tejidos blandos. En la consolidación puede tomar una forma irregular o engrosada y no lineal (Figura 2).

Signo del deslizamiento pleural (sliding sign): una vez identificada la línea pleural observamos un desplazamiento, que se describe como hallazgo dinámico entre la pleura parietal y la pleura visceral. Esta percepción de movimiento se genera durante cada ciclo respiratorio.

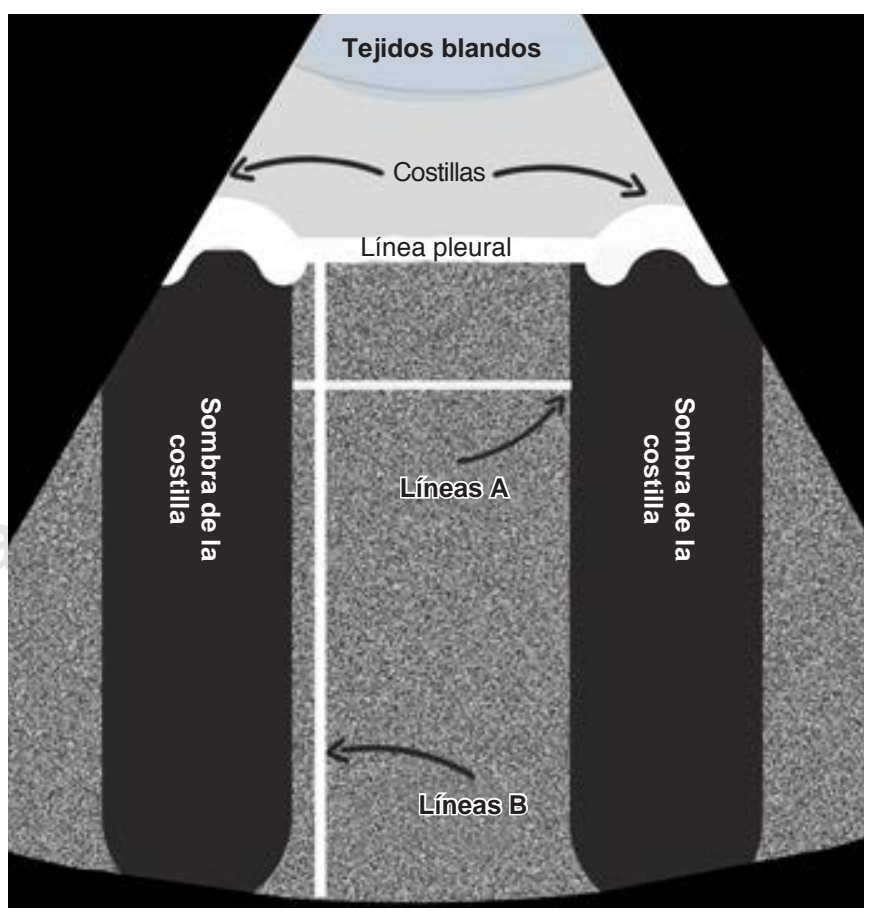

Figura 2: Artefactos en ultrasonido pulmonar. 


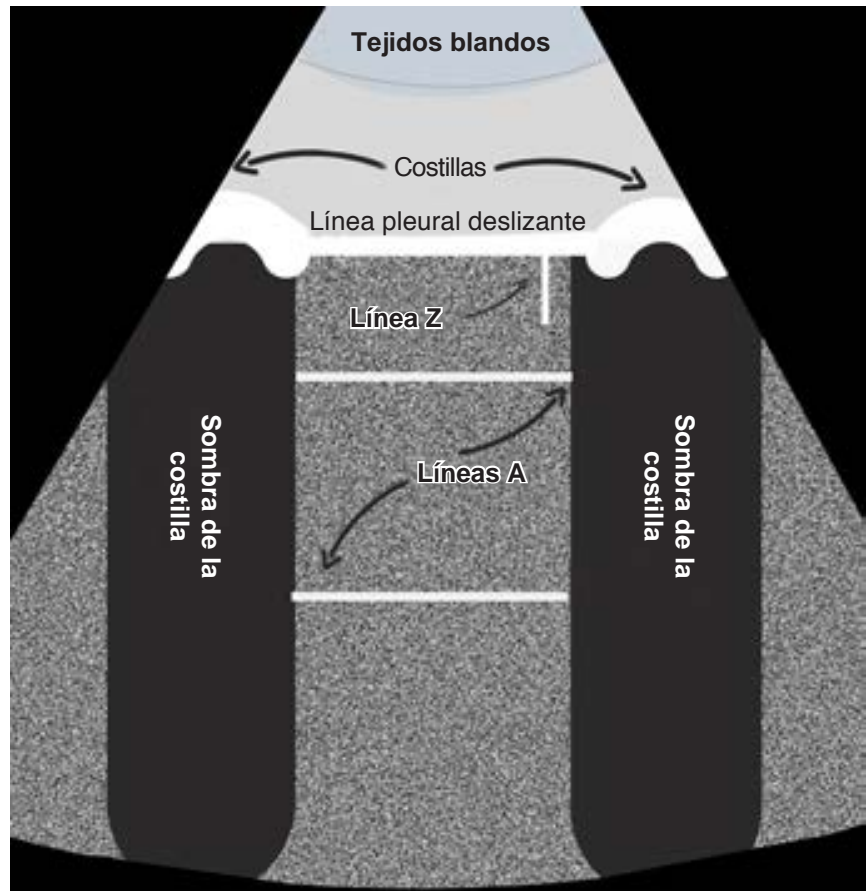

Figura 3: Etapa 0.

Líneas A. Son artefactos con forma de líneas horizontales, son hiperecogénicas, similares a la línea pleural, pero que se repiten de forma paralela y equidistante hasta el final de la pantalla. Su ecogenicidad puede atenuarse con la profundidad. Estas líneas indican la presencia de aire (pulmón aireado).

Líneas B. Son artefactos en forma de líneas perpendiculares a la pleura de donde se originan; como las líneas A son líneas también hiperecogénicas y generalmente bien definidas, se extienden siempre hacia el final de la pantalla, borran las líneas A y se movilizan con la línea pleural. Pueden observarse agrupadas $y /$ confluentes. ${ }^{18}$

\section{INTEGRACIÓN DE SIGNOS}

En la neumonía viral por SARS-CoV-2, se describen cuatro fases de progresión; cada fase se caracteriza por una pérdida progresiva de la aireación pulmonar. Aparentemente esto también sigue un patrón reversible en caso de recuperación, por lo que puede ser útil en la identificación del curso de la infección. Además, los hallazgos más severos (consolidación) parecen corresponder con deterioro clínico y, por lo tanto, pueden usarse para predecir el requerimiento de una mayor necesidad de soporte. Los patrones de ultrasonido pulmonar encontrados en pacientes con infecciones COVID-19 son también típicos de otra neumonía intersticial viral. ${ }^{24,25}$

Dividiremos en las siguientes cuatro etapas la progresión ultrasonográfica basándonos en el patrón de aire pulmonar:
Etapa 0. Bien ventilado (ausencia de síntomas o fase de recuperación) (Figura 3).

Para que el aspecto de la ecografía pulmonar se considere normal deben estar presentes tanto las líneas $A$, como el deslizamiento pleural del pulmón. La imagen profunda a la línea pleural en un pulmón normalmente aireado es un artefacto y no una imagen del parénquima pulmonar. El resto del artefacto aparece como una sombra moteada gris y cambia de apariencia durante la respiración, similar a la imagen que produce la estática de un televisor. Como la línea pleural es altamente reflectante, generalmente se duplica a continuación como artefactos de reverberación (líneas A). Hay que recordar que pequeños grados de manipulación en el transductor podría mejorar la apariencia de las líneas A. Como parte de los hallazgos de patrón normal se pueden observar artefactos cortos verticales de cola de cometa que se denomina líneas Z; éstas se distinguen de las líneas B por su corta proyección.

Etapa 1 (temprana). Inicia la pérdida de la ventilación (Figura 4).

Este patrón corresponde con un síndrome intersticial inicial. Recordemos que el síndrome intersticial no es exclusivo de una patología. Este patrón se observa en el edema agudo pulmonar, neumonitis, neumonía intersticial, enfermedad pulmonar parenquimatosa difusa y en el síndrome de dificultad respiratoria aguda. El síndrome intersticial también puede ser causado por una patología focal como la consolidación, atelectasia, neoplasia, entre otros. ${ }^{26}$ Lo que caracteriza al síndrome

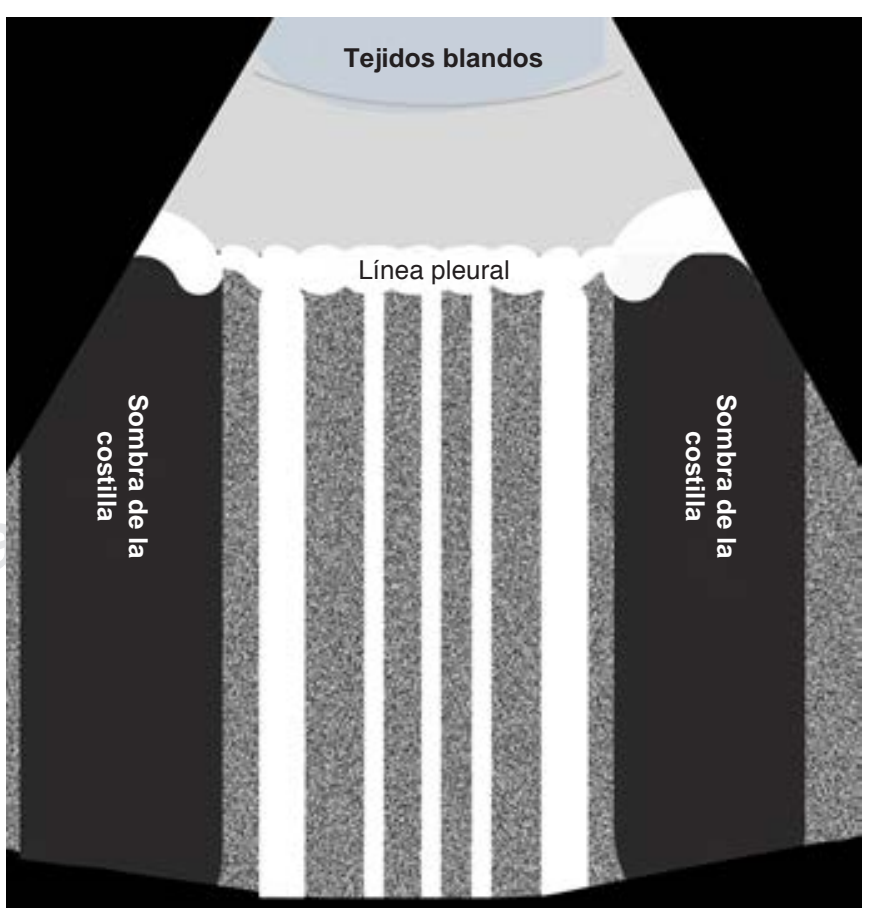

Figura 4: Etapa 1. 


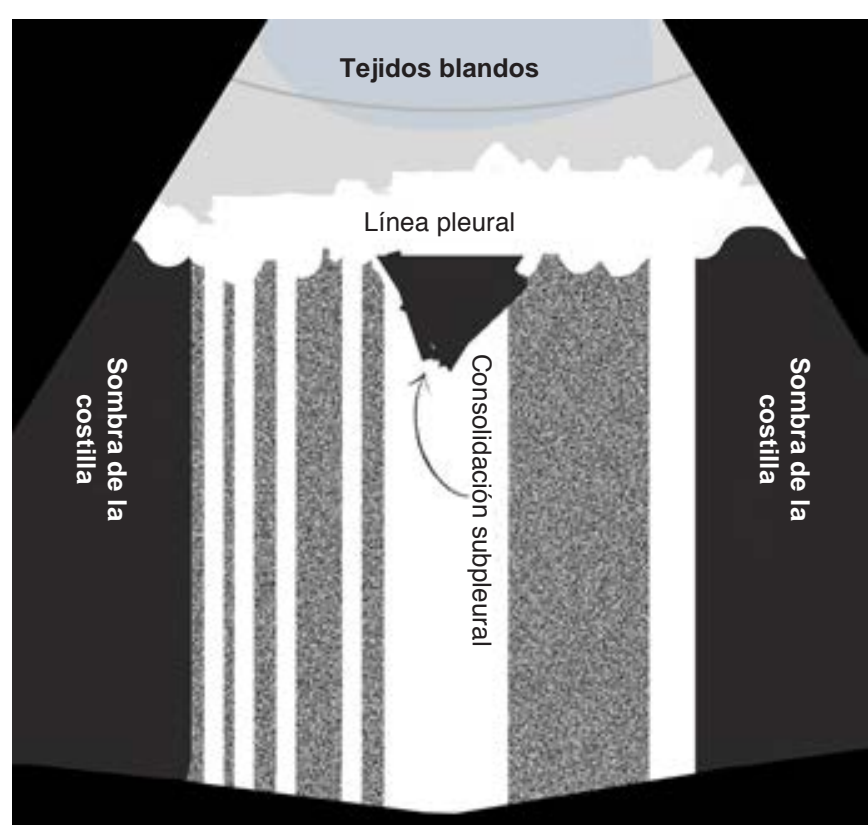

Figura 5: Etapa 2.

intersticial en el ultrasonido es la presencia de líneas B (mayor a 3). Recordemos que éstos son artefactos formados por la reverberación de la ecografía dentro del líquido y probablemente dentro de los tabiques interlobulares. Los diferentes trastornos que produce el síndrome pueden diferenciarse según la ubicación del patrón $B$, el carácter de las líneas $B$, la presencia de anomalías pleurales y la consolidación. Por lo tanto, en la etapa 1, se observan líneas B o el patrón B, el cual, a menudo no es homogéneo (líneas $B$ discretas sin coalescencia) con áreas libres, más comúnmente observadas en las zonas laterales, posteriores e inferiores. Hay deslizamiento pulmonar y las irregularidades pleurales no son marcadas.

Como la neumonitis por COVID-19 es típicamente bilateral (etapa 2), el síndrome intersticial localizado podría sugerir una fase temprana de la enfermedad (etapa 1). Existe reporte de disfunción cardiaca en el paciente con COVID-19. La ecografía pulmonar es superior y más sensible en el diagnóstico de edema agudo pulmonar que en la radiografía de tórax. ${ }^{27}$

El deslizamiento pulmonar se conserva normalmente, ya que la pleura no se ve afectada. El edema pulmonar incluye el patrón B en ambos pulmones, en las áreas adyacentes o sin áreas de interrupción y que, generalmente, son más prominentes en él (aumento de la densidad de las líneas B), también en las zonas posteroinferiores del pulmón (zonas dependientes) e incluso, en esta zona puede verse asociado el derrame pleural. El número de líneas $B$ se ha correlacionado proporcionalmente con la gravedad de la insuficiencia cardiaca aguda. ${ }^{28}$ Otra de las características es que las líneas
B aparecen y desaparecen en respuesta a la carga y eliminación de líquidos, incluso se puede utilizar para evaluar respuesta a los tratamientos. Por supuesto consideramos de vital importancia los hallazgos cardiacos y el contexto clínico del paciente para dar certeza. En resumen, la diferenciación de síndrome intersticial por COVID frente al edema agudo pulmonar cardiaco debe ser relativamente sencilla, puesto que las líneas $B$ suelen ser homogéneas, sin áreas libres y con mayor densidad en las zonas dependientes.

Etapa 2 (intermedia). Mínimamente aireado (Figura 5). A medida que la neumonitis progresa, hay más áreas con patrón $B$ y las líneas $B$ tienen una densidad aumentada y se vuelven confluentes (pulmón blanco) en algunas áreas La línea pleural continúa engrosándose y presentando irregularidades marcadas. Se puede observar engrosamiento y edema en los tejidos intersticiales. En esta etapa aparecen las consolidaciones subpleurales, las cuales pueden volverse múltiples de acuerdo con la progresión del COVID-19. El examen Doppler color de estas consolidaciones generalmente demuestra un flujo sanguíneo reducido.

Etapa 3 (avanzada) completa pérdida de la aireación. Etapa de consolidación pulmonar (Figura 6).

La identificación de una consolidación pulmonar es relativamente accesible, recordemos que como parte importante de la fisiopatología ocurre un llenado de líquido en los espacios alveolares pulmonares. Esto permite la transmisión más eficiente de la ecografía. La consolidación aparece en el ultrasonido como una ima-

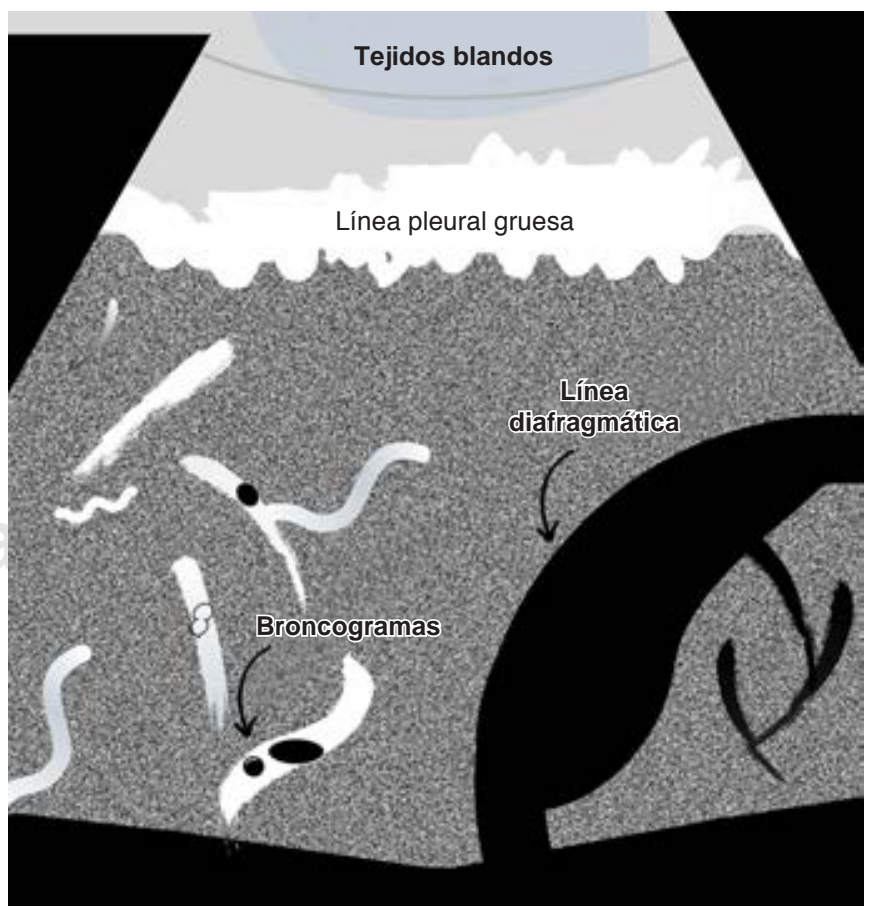

Figura 6: Etapa 3. 
gen que puede parecer un tejido y, por ello, se acuñó el término de (hepatización). Sin embargo, a diferencia del hígado, el pulmón consolidado comúnmente todavía contiene bronquios con paredes de cartílago y aire, vistos como puntos hiperecogénicos con líneas ramificadas. Se ha propuesto llamar a estos puntos broncogramas aéreos.

Es común que la consolidación y la atelectasia coexistan y a menudo es difícil discriminar entre ellas. El carácter de los broncogramas aéreos puede permitir la discriminación entre estas dos condiciones. ${ }^{29,30}$ Los broncogramas aéreos son resultado de una interfase aire-liquido, pues el aire se encuentra atrapado dentro de los bronquiolos llenos de líquido y puede observarse con la respiración (broncrogramas dinámicos). Cuando se encuentran, confirman el diagnóstico de consolidación.

Se han descrito broncrogamas estáticos (sin cambios con la respiración) como parte del espectro de la atelectasia. La atelectasia tiene una imagen muy similar a la consolidación, incluso pueden coexistir. La característica principal que distingue a la atelectasia de la consolidación es que en la atelectasia casi siempre se produce pérdida de volumen pulmonar. Generalmente se observan líneas hiperecogénicas lineales que se originan en un núcleo hiperecoico aún mayor, causadas por el aire atrapado dentro del pulmón colapsado. El deslizamiento pulmonar puede reducirse o aparentemente estar ausente con consolidación y atelectasia, debido a la expansión pulmonar reducida durante la respiración. La prueba definitiva que distingue la atelectasia de la consolidación es la demostración con ultrasonido de la reexpansión de las unidades pulmonares colapsadas después de una maniobra de reclutamiento.

\section{Precauciones con el equipo}

Se deben tomar todas las precauciones con la finalidad de evitar la contaminación del dispositivo y la transmisión del SARS-CoV-2. En general, se acepta que la ultrasonografía pulmonar es un procedimiento de bajo riesgo infeccioso, sin embargo, las medidas rigurosas de control son imprescindibles.

Se debe considerar antes de la realización del examen: higiene de manos, limpiar la consola y pantalla con agentes de alto nivel para instrumentos; lo anterior de acuerdo con la recomendación del fabricante. Se sugiere cubrir con una lámina de plástico al equipo y considere exclusivamente el uso de gel.

Durante el examen de ultrasonido se debe limitar el tiempo de exploración y la realización por el personal con mayor experiencia.

Después de realizar el examen toda la máquina debe asearse dos veces con agentes de limpieza de alto nivel para instrumentos. El primer momento es al pie de la cama del paciente, y el segundo momento lejos del paciente junto con EPP limpio, de preferencia por una segunda persona. En caso de contaminación directa de fluidos corporales del paciente o, si tiene dudas, se debe realizar una desinfección formal de «alto nivel» de acuerdo con los protocolos del hospital.

\section{CONCLUSIONES}

Durante la pandemia de COVID-19, la ultrasonografía pulmonar tiene un papel protagónico. El ultrasonido permite una evaluación no invasiva y constante. Su aplicación permitirá la identificación correcta de la gravedad, la evolución y recuperación pulmonar. Resaltamos la importancia del USP como pieza clave en el monitoreo de estos pacientes y señalamos también una reducción en el uso de radiografías de tórax y tomografías computarizadas, lo que permite la disminución del riesgo de exposición y aumenta la eficiencia en el manejo.

\section{BIBLIOGRAFÍA}

1. Munster VJ, Koopmans M, van Doremalen N, van Riel D, de Wit E. A novel coronavirus emerging in China-key questions for impact assessment. N Engl J Med. 2020;382(8):692-694.

2. Bavishi C, Maddox TM, Messerli FH. Coronavirus disease 2019 (COVID-19) infection and renin angiotensin system blockers. JAMA Cardiol. 2020. doi: 10.1001/jamacardio.2020.1282.

3. Huang C, Wang Y, Li X, Ren L, Zhao J, Hu Y, et al. Clinical features of patients infected with 2019 novel coronavirus in Wuhan, China. Lancet. 2020;395(10223):497-506.

4. Ryu S, Chun BC; Korean Society of Epidemiology 2019-nCoV Task Force Team. An interim review of the epidemiological characteristics of 2019 novel coronavirus. Epidemiol Health. 2020;42:e2020006.5.

5. Chung M, Bernheim A, Mei X, Zhang N, Huang M, Zeng X, et al. CT imaging features of 2019 novel coronavirus (2019-nCoV). Radiology. 2020;295(1):202-207.

6. Corradi F, Brusasco C, Pelosi P. Chest ultrasound in acute respiratory distress syndrome. Curr Opin Crit Care. 2014;20(1):98-103.

7. Raheja R, Brahmavar M, Joshi D, Raman D. Application of lung ultrasound in critical care setting: a review. Cureus. 2019;11(7):e5233.

8. Liccardo B, Martone F, Trambaiolo P, Severino S, Cibinel GA, D'Andrea A. Incremental value of thoracic ultrasound in intensive care units: Indications, uses, and applications. World J Radiol. 2016;8(5):460-471.

9. Lichtenstein D, van Hooland S, Elbers P, Malbrain ML. Ten good reasons to practice ultrasound in critical care. Anaesthesiol Intensive Ther. 2014;46(5):323-335.

10. Lumb P, Karakistios D. Critical care ultrasound. Philadelphia: Elsevier/Saunders; 2016.

11. Lichtenstein D, Goldstein I, Mourgeon E, Cluzel P, Grenier $P$, Rouby JJ. Comparative diagnostic performances of auscultation, chest radiography, and lung ultrasonography in acute respiratory distress syndrome. Anesthesiology. 2004;100(1):9-15.

12. Xirouchaki N, Magkanas E, Vaporidi K, Kondili E, Plataki M, Patrianakos $A$, et al. Lung ultrasound in critically ill patients: comparison with bedside chest radiography. Intensive Care Med. 2011;37(9):1488-1493.

13. Winkler MH, Touw HR, van de Ven PM, Twisk J, Tuinman PR. Diagnostic accuracy of chest radiograph, and when concomitantly studied lung ultrasound, in critically ill patients with respiratory symptoms: a systematic review and meta-analysis. Crit Care Med. 2018;46(7):e707-e714. 
14. Jankowich EM. Ultrasound in the Intensive Care Unit. New York: Springer Science; 2016. pp. 191-207.

15. Nazerian P, Volpicelli G, Vanni S, Gigli C, Betti L, Bartolucci M, et al. Accuracy of lung ultrasound for the diagnosis of consolidations when compared to chest computed tomography. Am J Emerg Med. 2015;33(5):620-625.

16. Tierney DM, Huelster JS, Overgaard JD, Plunkett MB, Boland LL, St Hill CA, et al. Comparative performance of pulmonary ultrasound, chest radiograph, and CT among patients with acute respiratory failure. Crit Care Med. 2020;48(2):151-157.

17. Lichtenstein D. Lung ultrasound in the critically ill: the BLUE protocol. Switzerland: Springer; 2016.

18. Mayo PH, Copetti R, Feller-Kopman D, et al. Thoracic ultrasonography: a narrative review. Intensive Care Med. 2019;45(9):1200-1211. doi: 10.1007/s00134-019-05725-8.

19. Volpicelli G, Elbarbary M, Blaivas M, et al. International evidencebased recommendations for point-of-care lung ultrasound. Intensive Care Med. 2012;38(4):577-591. doi: 10.1007/s00134012-2513-4.

20. Via G, Storti E, Gulati G, Neri L, Mojoli F, Braschi A. Lung ultrasound in the ICU: from diagnostic instrument to respiratory monitoring tool. Minerva Anestesiol. 2012;78(11):1282-1296.

21. Public Health England. Guidance COVID-19: epidemiology, virology and clinical features [sitio web]. Updated 27 March 2020 [acceso 29 de marzo 2020] Disponible en: https://www.gov.uk/ government/publications/wuhan-novel-coronavirus and-clinicalfeatures.

22. World Health Organization. Coronavirus disease 2019 (COVID-19) Situation Report-4 [sitio web]. [acceso 29 de marzo 2020] Disponible en: https://www.who.int/docs/default-source/ coronaviruse/situation-reports/20200301-sitrep-41-covid-19. pdf?sfvrsn $=6768306 d$ _ 2 .

23. Pan F, Ye T, Sun P, Gui S, Liang B, Li L, et al. Time course of lung changes at chest $\mathrm{CT}$ during recovery from coronavirus disease 2019 (COVID-19). Radiology. 2020;295(3):715-721.
24. Lichtenstein D, Mézière G, Biderman P, Gepner A, Barré O. The comet-tail artifact. An ultrasound sign of alveolar-interstitial syndrome. Am J Respir Crit Care Med. 1997;156(5):1640-1646.

25. Peng QY, Wang XT, Zhang LN; Chinese Critical Care Ultrasound Study Group (CCUSG). Findings of lung ultrasonography of novel corona virus pneumonia during the 2019-2020 epidemic. Intensive Care Med. 2020;46(5):849-850.

26. Picano E, Frassi F, Agricola E, Gligorova S, Gargani L, Mottola G. Ultrasound lung comets: a clinically useful sign of extravascular lung water. JAm Soc Echocardiogr. 2006;19(3):356-363.

27. Volpicelli G, Caramello V, Cardinale L, Mussa A, Bar F, Frascisco MF. Bedside ultrasound of the lung for the monitoring of acute decompensated heart failure. Am J Emerg Med. 2008;26(5):585-591.

28. Volpicelli G, Caramello V, Cardinale L, Cravino M. Diagnosis of radio-occult pulmonary conditions by real-time chest ultrasonography in patients with pleuritic pain. Ultrasound Med Biol. 2008;34(11):1717-1723.

29. Lichtenstein $D$, Mezière $G$, Seitz J. The dynamic air bronchogram. A lung ultrasound sign of alveolar consolidation ruling out atelectasis. Chest. 2009;135(6):1421-1425.

30. Reissig A, Kroegel C. Transthoracic ultrasound of lung and pleura in the diagnosis of pulmonary embolism: a novel noninvasive bedside approach. Respiration. 2003;70(5):441-452.

Correspondencia:

Francisco Godínez García

Hospital General de León.

Fracciones de los Aguirre, León, Guanajuato.

E-mail: dr.godinezfco@gmail.com 